\title{
Robust Model Predictive Control with One Free Control Move for NCSs with Data Missing
}

\author{
Jimin Yu \\ Key Laboratory of Industrial Internet of Things \\ \& Networked Control, Ministry of Education \\ Chongqing University of Posts and Telecom- \\ munications, Chongqing, PR China
}

\author{
Xiaogang Gong \\ Key Laboratory of Industrial Internet of Things \\ $\&$ Networked Control, Ministry of Education. \\ Chongqing University of Posts and Telecom- \\ munications, Chongqing, PR China
}

\begin{abstract}
Current hot issue of networked control systems(NCSs) remains how to optimize the signal transmission in imperfect links, especially, data missing(between the controller and actuator) is a potential source of poor performance of the control system. Here, stochastic variables satisfying markov jump process are used to describe the fading channel. Considering the uncertainty factor of plant, a practical compensation technique is utilized to minimize the effects caused by data dropout. Attention is paid to designing a useful control law to drive the closed-loop system stable and preserve a guaranteed infinite-horizon performance function, where the infinite-horizon control moves are parameterized as a free control move. Furthermore, the corresponding problems about recursive feasibility and stochastic stability are established by a set of linear matrix inequalities. Simulation results are shown to verify the performance of the proposed approach.
\end{abstract}

\section{General Terms}

Networked system, predictive control

\section{Keywords}

Data missing, polytoic model, state feedback.

\section{INTRODUCTION}

With the continuous advancements of communication technology, network has been inevitably induced in control zone because of its advantages, such as low cost, simple installation, maintenance and high reliability $([9],[8],[1],[10])$. Networked control systems are a class of feedback control system in structure, with control loops over networks for signals transmission, specifically, observations and feedback-control signals are transmitted through communication channels, computer can solve the complex optional algorithms, control theory lies in the core situation, respectively. However, considering the real time networked environment, the phenomenon of data loss are unavoidable due to the reasons of network congestion, limited bandwidth, etc [28]. Therefore, the issue about the optional performance and stability of NCSs becomes a hard work.

Research on data loss gets much attention. So far, many achievements have appeared. To mention a few. In [2], a self-triggered sampling scheme is proposed for a networked control system with consideration of data losses and communication delays, by making use of this scheme, the next sampling instant does not depend on on-line estimation of an event-triggered condition and the successive measurement of the state. Paper [17] studies the exponential synchronization of complex dynamical networks with control packet loss and additive time-varying delays, a novel LyapunovKrasovskii functional (LKF) with triple integral terms is constructed and by using Jensen's inequality and reciprocally convex approach, sufficient conditions under which the dynamical network is exponentially mean-square stable are derived. Considering both uniform and random consecutive data losses induced by long periods of transmission failure, a novel approach using a combination of prediction strategy and constant control gain based on gain error ratio (GER) is proposed for solving the problem by [20]. However, the above researches seldom use model predictive control method, which is an advanced optional algorithm.

Model predictive control(MPC) has a well-earned reputation in recent years, it provides on-line solutions to optimal feedback control problems, so model predictive control can be seen as a circular operation that a minimum problem is solved to calculated optional control inputs for a time horizon, specifically, at every instant, it requires the on-line solution of an optimization problem to compute optional control inputs. Although more than one control sequences are calculated, only the first one is implemented, at next instant, the optimization problem is reformulated and solved with new measurements obtained from the system [15]. Besides, another advantage of model predictive is that it can be conveniently used to solve the input and parameter constraints. In addition, several industrial process models as well as many performance index of importance can be handled by predictive control, for some references, the stable problems are studied in ([23], [14], [19]); the MPC for stochastic system is investigated in ([13], [6],[26], [25], [21]); for MPC of fuzzy system in ([29], [4], [11], [18], [16]).

Moreover, model predictive control shows the advantages in dealing with the model uncertainties, the problem of designing predictive controllers that explicitly optimize and stable the uncertainty system has obtained much interests from researchers and many great results have been made. see, e.g. Due to many of the robust model-predictive controllers developed to-date suffer from excessively conservative control, paper [12] presents a model predictive controller that uses a closed-loop model to estimate the uncertainty in future process inputs and outputs, the new controller solves a stochastic program at each execution in order to determine 
the set of control moves. A note ensured recursive feasibility in robust model predictive control with multiplicative uncertainty by applying terminal constraints to the degrees of freedom available to the controller while constructing polytopic tube cross sections, the technique in [24] by bounding the tube parameters in norm and constructing a terminal set based on this bound, which reduces the online computational load and so allows longer prediction horizons to be used.[7]propose an approach to design robust distributed model predictive control for polytopic uncertain networked control systems with time delays, An augmented polytopic uncertainty description is invoked to handle the input delays and the conservativeness of distributed predictive control algorithm is reduced by using a sequence of feedback control laws. In [27], robust model predictive control(RMPC) is compared with nominal predictive for the purposes of fore-aft tower damping of large wind turbines, the two controllers are identical save for their handling of the multiplicative and additive uncertainty in the prediction horizon.State and input constraints are applied to a control model that is identified by datadriven methods.

From above all, although, a great achievements on networked control systems have been made in recent years, however, how to solve the unreliable networks remains difficult and the problem of stability of stochastic system also brings obstacles to researches. This paper, therefore, focus on the stochastic networked system wherein controller outputs are transmitted via communication networks. Data loss inevitably happens in the communication link and stochastic variables satisfying the Markov process are used to describe the imperfect channel. Attention is devoted to derived a suitable controller such that the closed-loop system is stability and preserves a guaranteed optional performance.

\section{PROBLEM FORMULATION}

The structure of NCSs concerned in this paper is shown in fig 1

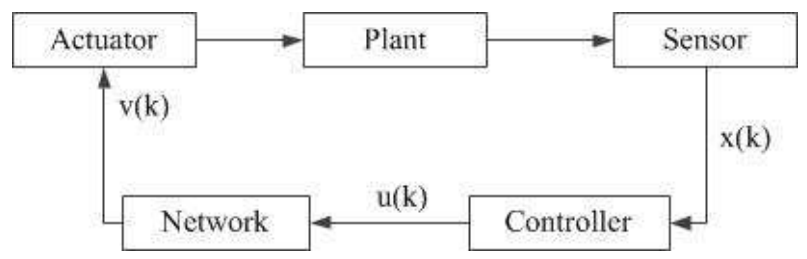

Fig. 1. The structure of networked control system(NCSs)

In fig 1. the plant, sensor, controller and actuator are spatially separated. $x(k) \in R^{n \times n}$ is the state vector, $u(k) \in R^{m \times m}$ is the control outputs and $v(k) \in R^{m \times m}$ is a signal received by actuator, and $k \geq 0$ is sampling time( or time step).

Now, the models of the physical plant, controller and transmission links are as follow.

Plant: the plant selected in this paper is a discrete linear time varying(LTV) system sampling periodically with $T>0$, which can be modeled as follow:

$$
\begin{aligned}
& x(k+1)=A(k) x(k)+B(k) v(k) \\
& y(k)=C x(k)
\end{aligned}
$$

Where the uncertainties are expressed by allowing the state-space matrices $A(k), B(k)$ to be arbitrarily time varying and lying in a polytope $[A(k) B(k)] \in \Theta=C o\left\{\left[A_{1} B_{1}\right], \cdots,\left[A_{l} B_{l}\right]\right\}$, and Co represents the convex hull, $\left[A_{i} B_{i}\right]$ means vertices of the convex hull and any $[A B]$ lying in $\Theta$ is a linear combinations of vertices
$[A(k) B(k)]=\sum_{i=0}^{l} \sigma_{i}\left[A_{i} B_{i}\right]$ for some non-negative $\sigma_{1}, \ldots, \sigma_{l}$ summing to one.

In this paper, the input constraints considered are the Euclidean norm constraints, that is, $v(k+i \mid k)^{T} v(k+i \mid k) \leq v_{\max }$.

Controller: Here a state-feedback control law is adopted.

$$
u(k)=K x(k) k \geq k_{0}
$$

Therefore, the corresponding predictive control law is:

$$
u(k+i \mid k)=K x(k+i \mid k) k \geq k_{0}, \quad i>0
$$

Communication links: communication links exist between the controller and actuator, according to assumption, the controller only sends data at each sampling time $k \geq 0$, the actuator only receives data at each sampling time $k \geq 0$. All data are transmitted in a single packet at each time. Because of the stochastic data loss, the control outputs are no longer equivalent to the inputs of the actuator, i.e $u(k) \neq v(k)$, to deal with this phenomena, a compensation strategy is as follow:

$$
v(k)=\lambda(k) u(k)+(1-\lambda(k)) \tau v(k-1)
$$

Where $\lambda(k) \in\{0,1\}$ is a stochastic variable modeling the imperfect nature of the link from the controller to the actuator, $\tau \in[0,1]$ is a forgetting factor selected by user. Assuming the data loss process is a discrete-time Markov process(failure and recovering), the corresponding probability is $P\{\lambda(k)=0 \mid \lambda(k)=$ $1\}=\alpha \in[0,1], P\{\lambda(k)=1 \mid \lambda(k)=0\}=\beta \in[0,1]$, respectively. According to the compensate strategy, at sampling time $\mathrm{k}$, if data are received, $v(k)=u(k)$, otherwise, $v(k)=\tau v(k-1)$, (zero-order hold effect).

By 1, 3, 3, defining $\bar{\eta}(k)=\left[x(k)^{T} \quad v(k-1)^{T}\right]^{T}$, where $\bar{\eta}(k) \in R^{m+n}$, according to all above equations, the model of closed-loop stochastic-networked system with time delays can be obtained.

$$
\bar{\eta}(k+1)=\psi(k)_{\lambda(k)} \bar{\eta}(k)
$$

where

$$
\psi(k)_{\lambda(k)}=\left[\begin{array}{cc}
A(k)+\lambda(k) B(k) K & (1-\lambda(k)) \tau B(k) \\
\lambda(k) K & (1-\lambda(k)) \tau I
\end{array}\right]
$$

Remark 1: For the closed-loop system(5), the uncertainties come from the self-structure properties of the plant and stochastic variable $\lambda(k)$. Considering the requirements of real-time of the physical plant, the sensor is clock-driven, controller and actuator are event-driven.

Remark 2: The paper assumes that data missing has an upper bound $h_{\max }$ (h successive loss), by the compensation strategy, the control inputs $\left\{v(k) \mid v(k)=\tau^{h} v(k-h), 0<h<h_{\max }\right\}$ stored in actuator buffer are applied to the system when failure transmission.

Now, the design about controller for the system (5) is shown as: Assuming that the system state is fully available, how to obtain a control law based on packet dropout such that the system (5) is stable and keep optional performance. 


\section{ROBUST MODEL PREDICTIVE CONTROL WITH WITH ONE FREE CONTROL MOVE FOR NCSS}

Given a common performance objective function as

$$
\min _{u(k+i)} \max _{A(k), B(k)} J_{0}^{\infty}(k)
$$

Where $J_{0}^{\infty}(k)=J_{0}^{1}(k)+J_{1}^{\infty}(k)$, specifically, first stage cost $J_{0}^{1}(k)=\bar{\eta}^{T}(k \mid k) S \bar{\eta}^{T}(k \mid k)+v^{T}(k \mid k) R v(k \mid k)$, and terminal cost $J_{1}^{\infty}(k)=\sum_{i=1}^{\infty}\left\{\bar{\eta}^{T}(k+i \mid k) S \bar{\eta}^{T}(k+i \mid k)+v^{T}(k+i \mid k) R v(k+\right.$ $i \mid k)\}$, here, $S=\operatorname{diag}\left\{S_{1}, 0\right\}, S_{1}>0$ and $R>0$ are reasonable weighting matrices.

Defining a quadratic Lyapunov function:

$$
\begin{array}{r}
V(x(k+i+1 \mid k), v(k+i \mid k))=x^{T}(k+i+1 \mid k) Q_{\lambda(k+i \mid k)} \times \\
x(k+i+1 \mid k)+v^{T}(k+i \mid k) G_{\lambda(k+i \mid k)} v(k+i \mid k)
\end{array}
$$

Satisfying $V(0)=0, \lambda(k+i)=w \in\{0,1\}$ and the following constraint:

$$
\begin{aligned}
& E\{V(k+i+1 \mid k)-V(k+i \mid k)\} \\
& \leq E\left\{\bar{\eta}(k+i \mid k)^{T} S \bar{\eta}(k+i \mid k)+v(k+i \mid k)^{T} R v(k+i \mid k)\right\}
\end{aligned}
$$

Summing up the inequality 8 from $i=1$ to $\infty$, for the quadratic function to be finite, taking $\lim _{i \rightarrow \infty} E\{\bar{\eta}(k+i \mid k)\}=0$, so $\lim _{i \rightarrow \infty} E\{V(k+i \mid k)\}=0$. Thus

$$
J_{1}^{\infty} \leq E\{V(k+1 \mid k)\}
$$

Let

$$
\begin{gathered}
x^{T}(k \mid k) S_{1} x(k \mid k)+v^{T}(k \mid k) R v(k \mid k)+x^{T}(k+1 \mid k) \times \\
Q_{\lambda(k+1 \mid k)} x(k+1 \mid k)+v^{T}(k \mid k) G_{\lambda(k \mid k)} v(k \mid k) \leq \gamma(k)
\end{gathered}
$$

Where $x(k \mid k)=x(k)$ is the state of the system at sampling instant $\mathrm{k}$. Therefore, the minimization of $J_{0}^{\infty}$ is converted to getting the minimization of a variable $\gamma$ subject to

$$
\begin{aligned}
& \min _{u(k+i)} \max _{A(k), B(k)} \gamma(k) \\
& \text { S.t [5, 6, (8), 9) }
\end{aligned}
$$

\subsection{Stochastic invariant constraints about system states}

Lemma1:(Stochastic Invariant ellipsoid) Following ([5], [3], [22]), the states are as follow.

$$
\begin{aligned}
& \bar{\eta}(k+i \mid k) \in \Omega=\left\{\bar{\eta} \mid \bar{\eta}^{T} \operatorname{diag}\left\{\bar{Q}_{1}^{-1}, \bar{G}_{1}^{-1}\right\} \bar{\eta} \leq 1\right\} \\
& \lambda(k+i \mid k)=1 \text { and } V(k+i \mid k) \leq \gamma
\end{aligned}
$$

If there is a scalar $\gamma(\gamma>0)$, symmetric matrices $\bar{Q}_{1}, \bar{G}_{1}, \bar{Q}_{0, s}, \bar{G}_{0, s},\left(s=1, \ldots, h_{\max }\right)$ and Y satisfy the following LMIs with $i=1, \cdots l$ :

$$
\left[\begin{array}{cccc}
\bar{Q}_{1} & * & * & * \\
0 & \bar{G}_{1} & * & * \\
A_{i} \bar{Q}_{1}+B_{i} Y & 0 & \bar{Q}_{1} & * \\
Y & 0 & 0 & \bar{G}_{1}
\end{array}\right] \geq 0
$$

$$
\left[\begin{array}{cccc}
\bar{Q}_{1} & * & * & * \\
0 & \bar{G}_{1} & * & * \\
A_{i} \bar{Q}_{1}+B_{i} Y & 0 & \bar{Q}_{0,1} & * \\
Y & 0 & 0 & \bar{G}_{0,1}
\end{array}\right] \geq 0
$$

$$
\begin{aligned}
& {\left[\begin{array}{cccc}
\bar{Q}_{0, s} & * & * & * \\
0 & \bar{G}_{0, s} & * & * \\
A_{i} \bar{Q}_{0, s} & \tau B_{i} \bar{G}_{0, s} & \bar{Q}_{1} & * \\
0 & \tau \bar{G}_{0, s} & 0 & \bar{G}_{1}
\end{array}\right] \geq 0} \\
& s=1, \ldots, h_{\max } \\
& \left.\begin{array}{cccc}
\bar{Q}_{0, s} & \bar{G}_{0, s} & * & * \\
0 & * & * \\
A_{i} \bar{Q}_{0, s} & \tau B_{i} \bar{G}_{0, s} & \bar{Q}_{0, s} & * \\
0 & \tau \bar{G}_{0, s+1} & 0 & \bar{G}_{0, s+1}
\end{array}\right] \geq 0
\end{aligned}
$$

In this case, $\zeta=\left\{\bar{\eta} \mid \bar{\eta}^{T} \operatorname{diag}\left\{\bar{Q}_{1}, \bar{G}_{1}\right\} \bar{\eta}\right\}$ is an invariant ellipsoid for the predicted states of the uncertainty system with a desired control gain K satisfying $K=Y \bar{Q}_{1}^{-1}$.

Proof: By assumption, the solution from optional performance problem can be transmitted successively at time $\mathrm{k}$, based on the model $(5), \bar{\eta}(k+1 \mid k)=\Psi(k)_{1} \bar{\eta}(k \mid k)$. At time $\mathrm{k}+1$, the paper only considers the case $1 \rightarrow 1$, that is, $\lambda(k \mid k)=1, \lambda(k+1 \mid k)=1$, if 12. satisfied, thus $\bar{\eta}(k+1 \mid k)^{T} \operatorname{diag}\left\{\bar{Q}_{1}^{-1}, \bar{G}_{1}^{-1}\right\} \bar{\eta}(k+1 \mid k)^{T} \leq$ $1\}$, which means $\bar{\eta}(k+1 \mid k) \in \Omega$ for $\lambda(k+1 \mid k)=1$.

At time $\mathrm{k}+2$, two cases will be taken into account for $\lambda(k+2 \mid k)=$ 1. (i) $1 \rightarrow 1 \rightarrow 1$,i.e, $\lambda(k \mid k)=1, \lambda(k+1 \mid k)=1, \lambda(k+2 \mid k)=1$, using (12), it's easily get $\bar{\eta}(k+2 \mid k) \in \Omega$; (ii) $1 \rightarrow 0 \rightarrow 1$, i.e, $\lambda(k \mid k)=1, \lambda(k+1 \mid k)=0, \lambda(k+2 \mid k)=1$, we define $\bar{\eta}(k+1 \mid k) \in \Omega_{0,1}:=\left\{\bar{\eta} \mid \bar{\eta}^{T} \operatorname{diag}\left\{\bar{Q}_{0,1}, \bar{G}_{0,1}\right\} \bar{\eta}\right\}$. So only $\sqrt{13}$, (14) hold, we have

$$
\begin{aligned}
& \bar{\eta}(k+2 \mid k)^{T} \operatorname{diag}\left\{\bar{Q}_{0,1}, \bar{G}_{0,1}\right\} \bar{\eta}(k+2 \mid k) \leq \bar{\eta}(k+1 \mid k)^{T} \times \\
& \operatorname{diag}\left\{\bar{Q}_{1}, \bar{G}_{1}\right\} \bar{\eta}(k+1 \mid k) \leq 1
\end{aligned}
$$

Then $\bar{\eta}(k+2 \mid k) \in \Omega$

As for time $k+i(i \geq 3)$, the following conclusions are obtained for $\lambda(k+s \mid k)=1$ : (i) $\lambda(k+s \mid k)=1, s=1,2,3, \ldots$, using (12), it is easily to get $\bar{\eta}(k+s \mid k) \in \Omega$; (ii) $\lambda(k \mid k)=1, \lambda\left(k+h_{\text {max }} \mid k\right)=$ $1, \lambda(k+s \mid k)=0\left(1<s<h_{\max }\right)$, we also define $\bar{\eta}(k+s \mid k) \in$ $\Omega_{0, s}:=\left\{\bar{\eta} \mid \bar{\eta}^{T} \operatorname{diag}\left\{\bar{Q}_{0, s}, \bar{G}_{0, s}\right\} \bar{\eta}\right\},\left(1<s<h_{\max }\right)$,if $\left.\sqrt{13}\right)$, (14), 15 hold, it concludes $\bar{\eta}(k+s \mid k) \in \Omega$ after h successive delays. Proof end.

\subsection{Predictive control law design for stochastic networked system}

In this subsection, the design of controller will be given, which drives the stochastic system stable and minimizes the value of the objective function. The following theorems shows a procedure which converts the controller design to a solution of an convex optimization problem.

Theorem1:Let $x(k)=x(k \mid k)$ to be the state of stochastic uncertainty system measured at time $\mathrm{k}$ and there are not any constraints on the inputs. Supposing the communication link parameter $\alpha$ and $\beta=1-\alpha$ are given stochastically(satisfy $0 \leq \alpha \leq 1,0 \leq \beta \leq 1$ ) and the matrices $S>0, R>0,0 \leq \tau \leq 1$. For the structure uncertainty system, suppose there exists a state feedback control law 
such that the worst case MPC objective function $J_{0}^{\infty}$ is minimized if there exist matrix $\bar{Q}_{0}, \bar{Q}_{1}, \quad, \bar{G}_{0}, \bar{G}_{1}, \bar{W}_{0}, \bar{W}_{1}, \quad \bar{T}_{0}, \bar{T}_{1}$ and $\mathrm{Y}$, such that the below convex minimization problem is feasible

$$
\min \gamma
$$

Subject to the following inequalities with $i=1, \cdots, l$

$$
\left[\begin{array}{ccccc}
1 & * & * & * & * \\
T(k) & \bar{Q}_{1} & * & * & * \\
v(k) & 0 & \bar{G}_{1} & * & * \\
S_{1}^{1 / 2} x(k) & 0 & 0 & \gamma I & * \\
R^{1 / 2} v(k) & 0 & 0 & 0 & \gamma I
\end{array}\right] \geq 0
$$

$$
\left[\begin{array}{cccccc}
\bar{Q}_{1} & * & * & * & * & * \\
0 & \bar{G}_{1} & * & * & * & * \\
A_{i} \bar{Q}_{1}+B_{i} Y & 0 & \bar{W}_{1} & * & * & * \\
Y & 0 & 0 & \bar{T}_{1} & * & * \\
\bar{Q}_{1}^{T} & 0 & 0 & 0 & \gamma S_{1}^{-1} & * \\
Y & 0 & 0 & 0 & 0 & \gamma R^{-1}
\end{array}\right] \geq 0
$$

$$
\left[\begin{array}{cccccc}
\bar{Q}_{0} & * & * & * & * & * \\
0 & \bar{G}_{0} & * & * & * & * \\
A_{i} \bar{Q}_{0}^{T} & \tau B_{i} \bar{G}_{0}^{T} & \bar{W}_{0} & * & * & * \\
0 & \tau \bar{G}_{0}^{T} & 0 & \bar{T}_{0} & * & * \\
\bar{Q}_{0}^{T} & 0 & 0 & 0 & \gamma S_{1}^{-1} & * \\
0 & \tau \bar{G}_{0}^{T} & 0 & 0 & 0 & \gamma R^{-1}
\end{array}\right] \geq 0
$$

$$
\left[\begin{array}{cccccc}
\bar{W}_{1} & * & * & * & * & * \\
0 & \bar{T}_{1} & * & * & * & * \\
\alpha^{1 / 2} \bar{W}_{1}^{T} & 0 & \bar{Q}_{0} & * & * & * \\
(1-\alpha)^{1 / 2} \bar{W}_{1}^{T} & 0 & 0 & \bar{Q}_{1} & * & * \\
0 & \alpha^{1 / 2} \bar{T}_{1}^{T} & 0 & 0 & \bar{G}_{0} & * \\
0 & (1-\alpha)^{1 / 2} \bar{T}_{1}^{T} & 0 & 0 & 0 & \bar{G}_{1}
\end{array}\right] \geq 0
$$

$$
\left[\begin{array}{cccccc}
\bar{W}_{0} & * & * & * & * & * \\
0 & \bar{T}_{0} & * & * & * & * \\
(1-\beta)^{1 / 2} \bar{W}_{0}^{T} & 0 & \bar{Q}_{0} & * & * & * \\
\beta^{1 / 2} \bar{W}_{0}^{T} & 0 & 0 & \bar{Q}_{1} & * & * \\
0 & (1-\beta)^{1 / 2} \bar{T}_{0}^{T} & 0 & 0 & \bar{G}_{0} & * \\
0 & \beta^{1 / 2} \bar{T}_{0}^{T} & 0 & 0 & 0 & \bar{G}_{1}
\end{array}\right] \geq 0
$$

Where $T(k)=A_{i} x(k)+B_{i} v(k)$, if the above the minimization problem is feasible, the gain matrices of the predictive controller are given by

$$
K=Y \bar{Q}_{1}^{-1}
$$

Proof: Based on the compensation strategy, if data dropouts at time $\mathrm{k}$, the control law that has been calculated at previous time is used. In fact, the control law at time $\mathrm{k}$ is assumed to be transmitted successfully, the quadratic function $\mathrm{V}$ that we choose is (7) with $\bar{Q}_{1}=\gamma Q_{1}^{-1}, \bar{G}_{1}=\gamma G_{1}^{-1}$, using Schur complements, 9 is equal to 18 .
Also from the augmented model and the quadratic Lyapunov function, the contractiveness condition can be written as:

$$
\begin{aligned}
& E_{\bar{\eta}(k)}\left\{E_{\bar{\eta}(k+i \mid k)}\left[\bar{\eta}(k+i \mid k)^{T} \Psi^{T} \Phi_{\lambda(k+i+1 \mid k)} \Psi \bar{\eta}(k+i \mid k)\right]-\right. \\
& \left.\bar{\eta}(k+i \mid k)^{T} \Phi_{\lambda(k+i \mid k)} \bar{\eta}(k+i \mid k)\right\}+E_{\bar{\eta}(k)}\left\{\bar{\eta}(k+i \mid k)^{T} \times\right. \\
& \left.S \bar{\eta}(k+i \mid k)+v(k+i \mid k)^{T} R v(k+i \mid k)\right\} \leq 0
\end{aligned}
$$

Where $\Psi$ taking

$$
\left[\begin{array}{cc}
A(k+i)+\lambda(k+i \mid k) B(k+i) K(1-\lambda(k+i \mid k)) \tau B(k+i) \\
\lambda(k+i \mid k) K & (1-\lambda(k+i \mid k)) \tau I
\end{array}\right]
$$

$\Phi_{\lambda(k+s \mid k)}=\operatorname{diag}\left\{Q_{\lambda(k+s \mid k)}, G_{\lambda(k+s \mid k)}\right\}$.

$\Phi_{\lambda(k+i+1 \mid k)}=\operatorname{diag}\left\{Q_{\lambda(k+i+1 \mid k)}, \quad G_{\lambda(k+i+1 \mid k)}\right\}$. When $\lambda(k+i \mid k)=1$, inducing the transition matrix $\mathrm{P}$, we have $E_{\lambda(k+i \mid k)=1}\left\{\Phi_{\lambda(k+i+1 \mid k)}\right\}=\alpha \operatorname{diag}\left\{Q_{0}, G_{0}\right\}+(1-$ $\alpha) \operatorname{diag}\left\{Q_{1}, G_{1}\right\}$, so the 23 is satisfied if the following inequalities hold,

$$
\begin{aligned}
& {\left[\begin{array}{cc}
A(k+i)+B(k+i) & 0 \\
K & 0
\end{array}\right]^{T}\left[\begin{array}{cc}
W_{1} & 0 \\
0 & T_{1}
\end{array}\right]\left[\begin{array}{cc}
A(k+i)+B(k+i) & 0 \\
K & 0
\end{array}\right]} \\
& -\operatorname{diag} Q_{1}, G_{1}+\operatorname{diag} S_{1}, 0+\left[\begin{array}{ll}
K & 0
\end{array}\right]^{T} R\left[\begin{array}{ll}
K & 0
\end{array}\right] \leq 0 \\
& \alpha \operatorname{diag}\left\{Q_{0}, G_{0}\right\}+(1-\alpha) \operatorname{diag}\left\{Q_{1}, G_{1}\right\} \leq \operatorname{diag}\left\{W_{1}, T_{1}\right\}
\end{aligned}
$$

Pre- and post -multiplying 25, 26 using $\operatorname{diag}\left\{\gamma^{1 / 2} Q_{1}^{-1}, \gamma^{1 / 2} G_{1}^{-1}\right\}$ and $\operatorname{diag}\left\{\bar{W}_{1}^{-1}, T_{1}^{-\frac{1}{1}}\right\}$, respectively. Using schur complements with $\gamma Q_{1}^{-1}=\bar{Q}_{1}, \gamma G_{1}^{-1}=$ $\bar{G}_{1}, \gamma Q_{0}^{-1}=\bar{Q}_{0}, \gamma G_{0}^{-1}=\bar{G}_{0} \gamma W_{1}^{-1}=\bar{W}_{1}, \gamma T_{1}^{-1}=\bar{T}_{1}, Y=$ $K \bar{Q}_{1}$, we can get $(18)$ and 20). When $\lambda(k+s \mid k)=0$, by the similar procedure using $\gamma W_{0}^{-1}=\bar{W}_{0}, \gamma T_{0}^{-1}=\bar{T}_{0}$, we get 19 and 21 . proof end.

As for the input constraints condition:we have $\max _{s \geq 0} \| v(k+$ $s \mid k)\left\|_{2}^{2}=\max _{s>0}\right\| K x(k+s \mid k)\left\|_{2}^{2} \leq \max _{x^{T} x \leq 1}\right\| Y \bar{Q}_{1}^{-1 / 2} x \|_{2}^{2}=$ $\kappa_{\max }\left(\bar{Q}_{1}^{-1 / 2} Y^{T} Y Q_{1}^{-1 / 2}\right) \leq u_{\max }$, using schur complements,we get 27].

$$
\left[\begin{array}{cc}
u_{\max } & Y \\
Y^{T} & \bar{Q}_{1}
\end{array}\right] \leq 0
$$

Finally, summarizing an LMIs solution as:

$$
\begin{aligned}
& \min _{u(k \mid k), \Gamma, Y} \gamma(k) \\
& \text { s.t (5), 12 - 15, 17 - 21) and 27 }
\end{aligned}
$$

where $\Gamma$ taking $\left\{\bar{Q}_{0}, \bar{G}_{0}, \bar{Q}_{1}, \bar{G}_{1}, \bar{W}_{0}, \bar{T}_{0}, \bar{W}_{1}, \bar{T}_{1}, \bar{Q}_{01}, \bar{G}_{01}\right.$, $\left.\bar{Q}_{02}, \bar{G}_{02}, \bar{Q}_{03}, \bar{G}_{03}\right\}$.

\subsection{Recursive feasibility and stochastic stability of MPC}

Theorem2: The uncertainty model is (1) and (5), for initial time $k=k_{0}$, it supposes that the control inputs are transmitted from 
the controller to the actuator successfully and the optimization problem $(10)$ is feasible. Then the optimization problem is feasible for future time $k \geq k_{0}$.

Proof:Based on the assumption, the MPC optimization problem is feasible at initial time $k=k_{0}$ and $\lambda\left(k_{0}\right)=1$, the only LMI in the problem that influenced explicitly by the measured state $\bar{\eta}(k \mid k)=\bar{\eta}(k)$ of the system is 17). Therefore, to prove the recursive feasibility, it only proves (17) is feasibility for all future states $\bar{\eta}(k+i \mid k),(i>0)$.

Assuming that the optional solution in Theorem2 at time $k_{0}$ is $\left\{\gamma^{*}\left(k_{0}\right) \mid V\left(k_{0} \mid k_{0}\right) \leq \gamma^{*}\left(k_{0}\right)\right\}$, an optional solution $\left\{\gamma^{*}\left(k_{0}\right)\right\}$ is constructed at time $k=k_{0}+1$. Using lemma1, $V\left(k_{0}+1 \mid k_{0}\right) \leq \gamma^{*}\left(k_{0}\right)$, since the independent state measured at time $k_{0}+1$, that is, $\bar{\eta}\left(k_{0}+1 \mid k_{0}+1\right)=\bar{\eta}\left(k_{0}+1\right)$, equals $\bar{\eta}\left(k_{0}+1 \mid k_{0}\right)$, for any $[A(k) B(k)] \in \Theta$, which must satisfy $V\left(k_{0}+1 \mid k_{0}+1\right)=V\left(k_{0}+1 \mid k_{0}\right) \leq \gamma^{*}\left(k_{0}\right)$, thus the feasible solution at time $k_{0}$ is feasible at time $k_{0}+1$.

At time $k_{0}+2$, two cases will be considered: (i) $\lambda(k)=1, \lambda(k+$ $1)=1$, which means no delays.It assumes that the optional solution at time $k_{0}+1$ is $\left\{\gamma^{*}\left(k_{0}+1\right) \mid V\left(k_{0}+1 \mid k_{0}+1\right) \leq \gamma^{*}\left(k_{0}+1\right)\right\}$, using similar analysis at time $k_{0}+1,\left\{\gamma^{*}\left(k_{0}+1\right)\right\}$ is also feasible at time $k_{0}+2$. (ii) $\lambda(k)=1, \lambda(k+1)=0$, which denotes delays. Since the data delays at time $k_{0}+1$, the control input can not be transmitted to the actuator accurately, according to the compensation strategy, the control input at time $k_{0}$ stored in actuator buffer will be applied, so the optional solution $\left\{\gamma^{*}\left(k_{0}\right) \mid V\left(k_{0} \mid k_{0}\right) \leq \gamma^{*}\left(k_{0}\right)\right\}$ is constructed at time $k_{0}+2$ and $V\left(k_{0}+2 \mid k_{0}\right) \leq \gamma^{*}\left(k_{0}\right)$, Moreover, in such case, the independent state $\bar{\eta}\left(k_{0}+2 \mid k_{0}+2\right)=\bar{\eta}\left(k_{0}+2 \mid k_{0}\right)$ satisfy the state space equation. Thus it can be derived $V\left(k_{0}+2 \mid k_{0}+2\right)=V\left(k_{0}+2 \mid k_{0}\right) \leq \gamma^{*}\left(k_{0}\right)$, that is, $\left\{\gamma^{*}\left(k_{0}\right)\right\}$ is also feasible at time $k_{0}+2$.

As for $k_{0}+i(i \geq 3)$, similar conclusions can be derived in the same way.

Theorem3: For the model in (1) and (5), this paper proves the stability of polytopic system and it will refer the previous Lyapunov function $V(k+i \mid k)=\bar{\eta}(k+i \mid k)^{T} L_{k+i} \bar{\eta}(k+i \mid k)$, where $L_{k+i}=\operatorname{diag}\left\{Q_{\lambda(k+i \mid k)}, G_{\lambda(k+i \mid k)}\right\}>0$ is obtained from optional problem at time $k_{0}+i$. Further, the function $\mathrm{V}$ is a strictly decreasing. Considering the real time network with data loss, the optional solution at time $k_{0}$ is solved in Theorem 2 and control sets can be transmitted successfully, due to the recursive feasibility, the optional problem is feasibility for $k>k_{0}$. Moreover, the convex optimization has a unique minimum and a set of optional solution $\left(\gamma^{*}(k), L_{k}\right)$ at $k>k_{0}$.

At time $k_{0}+1$, for $\lambda\left(k_{0}+1\right)=0$, which means the optional solution $\left\{\gamma^{*}\left(k_{0}+1\right)\right\}$ failure. According to the compensation strategy, the input at time $k_{0}+1$ is dependent on $\left\{\gamma^{*}\left(k_{0}\right)\right\}$, then upper bound of $\gamma\left(k_{0}+1\right)$ is $\gamma^{*}\left(k_{0}\right)$. As for $\lambda\left(k_{0}+1\right)=1$, the optional results from Theorem 2 can be transmitted successfully, so

$$
\begin{aligned}
& \bar{\eta}\left(k_{0}+1 \mid k_{0}+1\right)^{T} L_{k_{0}+1} \bar{\eta}\left(k_{0}+1 \mid k_{0}+1\right) \leq \\
& \bar{\eta}\left(k_{0}+1 \mid k_{0}+1\right)^{T} L_{k_{0}} \bar{\eta}\left(k_{0}+1 \mid k_{0}+1\right)
\end{aligned}
$$

where $L_{k_{0}+1}$ is obtained from Theorem2, whereas $L_{k_{0}}$ is only feasible at time $k_{0}+1$. According to the predictive control law $u\left(k_{0}+s \mid k_{0}\right)=K \bar{\eta}\left(k_{0}+s \mid k_{0}\right)$ and Lemma1, for [A(k) B(k)] lying in polytope, we have

$$
\bar{\eta}\left(k_{0}+1 \mid k_{0}\right)^{T} L_{k_{0}} \bar{\eta}\left(k_{0}+1 \mid k_{0}\right) \leq \bar{\eta}\left(k_{0} \mid k_{0}\right)^{T} L_{k_{0}} \bar{\eta}\left(k_{0} \mid k_{0}\right)
$$

Because the independent state $\bar{\eta}\left(k_{0}+1 \mid k_{0}+1\right)=\bar{\eta}\left(k_{0}+1\right)$ equals $(A(k)+B(k) K) \bar{\eta}(k \mid k)$, which must satisfy 29). From 28) and (29), we conclude

$$
\begin{aligned}
& \bar{\eta}\left(k_{0}+1 \mid k_{0}+1\right)^{T} L_{k_{0}+1} \bar{\eta}\left(k_{0}+1 \mid k_{0}+1\right) \leq \\
& \bar{\eta}\left(k_{0} \mid k_{0}\right)^{T} L_{k_{0}} \bar{\eta}\left(k_{0} \mid k_{0}\right)
\end{aligned}
$$

Therefore, $\bar{\eta}(k \mid k)^{T} L_{k} \bar{\eta}(k \mid k)$ is decreasing function, in another words, $\gamma(k)$ is decreasing with $\mathrm{k}$, that is, $\gamma^{*}\left(k_{0}+1\right) \leq \gamma^{*}\left(k_{0}\right)$. At for time $k_{0}+2$, based on the above similar analysis, the following conclusions can be obtained:

(i)for $\lambda\left(k_{0}+1\right)=0, \lambda\left(k_{0}+2\right)=0$, from Lemma1, the upper bound of $\gamma\left(k_{0}+2\right)$ is $\gamma^{*}\left(k_{0}\right)$;

(ii)for $\lambda\left(k_{0}+1\right)=0, \lambda\left(k_{0}+2\right)=1$, based on the optimization principle, we have $\gamma\left(k_{0}+2\right) \leq \gamma\left(k_{0}\right)=\gamma^{*}\left(k_{0}\right)$;

(iii)for $\lambda\left(k_{0}+1\right)=1, \lambda\left(k_{0}+2\right)=0$, from Lemma1 the upper bound of option value $\gamma\left(k_{0}+2\right)$ is $\gamma^{*}\left(k_{0}+1\right) \leq \gamma^{*}\left(k_{0}\right)$;

(iiii) for $\lambda\left(k_{0}+1\right)=1, \lambda\left(k_{0}+2\right)=1$,based on the optimization principle $\gamma\left(k_{0}+2\right)=\gamma^{*}\left(k_{0}+2\right) \leq \bar{\gamma}\left(k_{0}+1\right)=\gamma^{*}\left(k_{0}+1\right) \leq$ $\gamma^{*}\left(k_{0}\right)$;

As for time $k_{0}+i(i \geq 3)$, similar results can be obtained. Specifically, if there exist $\mathrm{h}$ successive data time-delay, the upper bound of $\left\{\gamma^{*}\left(k_{0}+i\right)\right\}$ is $\left\{\gamma^{*}\left(k_{0}+i-h\right)\right\}$, if transmitted successfully, $\gamma\left(k_{0}+i\right)=\gamma^{*}\left(k_{0}+i\right) \leq=\gamma^{*}\left(k_{0}+i-1\right)$, because the maximum data time-delay is bounded by $h_{\max }$, so $\left\{\gamma\left(k_{0}\right)\right\}$ is decreasing with $\mathrm{k}$. According to the contractiveness condition, the following equation can be obtained.

$$
\begin{aligned}
& \sum_{i=0}^{\infty} E_{\bar{\eta}\left(k_{0}\right)}\left\{\bar{\eta}(k+i \mid k)^{T} S \bar{\eta}(k+i \mid k)+v(k+i \mid k)^{T} R \times\right. \\
& v(k+i \mid k)\} \leq \gamma^{*}\left(k_{0}\right)
\end{aligned}
$$

Without loss of generality,it takes $k_{0}=0$, from above

$$
\sum_{i=0}^{\infty} E_{\bar{\eta}(0)}\left\{\bar{\eta}(k+i \mid k)^{T} S \bar{\eta}(k+i \mid k)\right\} \leq \infty
$$

Let $z=\lambda_{\min }\left(S_{1}\right)$,so

$$
\begin{aligned}
& E_{\bar{\eta}(0)} \Sigma_{k=0}^{\infty}\left\{\bar{\eta}(k)^{T} S \bar{\eta}(k)\right\} \leq(1 / z) \\
& E_{\bar{\eta}(0)} \Sigma_{k=0}^{\infty}\left\{\bar{\eta}(k)^{T} S \bar{\eta}(k)\right\}<\infty
\end{aligned}
$$

So the stochastic stable of closed-loop system is proved from [30].

\section{ILLUSTRATE EXAMPLE}

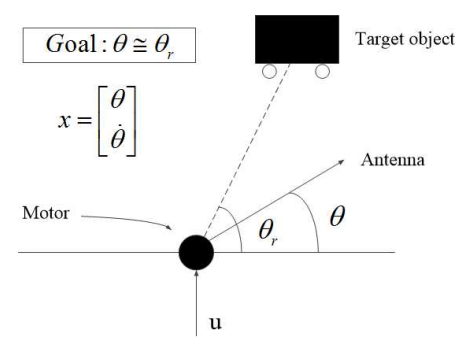

Fig. 2. Angular positioning system

In this section, a classical angular positioning system (fig 2 is used to prove the effectiveness of proposed design. The system consists of a rotating antenna at the origin of the plane,driven by an electric motor,the aim is to find the input voltage to the motor to rotate the antenna such that it points in the direction of a moving object in the plane. Defining $x(k)=\left[\begin{array}{lll}x_{1}^{T}(k) & x_{2}^{T}(k)\end{array}\right]^{T}=\left[\begin{array}{ll}\theta^{T}(k) & \dot{\theta}^{T}(k)\end{array}\right]^{T}$ and 
sampling time $T=0.1 s$, the closed-loop model can be written as follow:

$$
x(k+1)=A(k) x(k)+B(k) v(k)
$$

Where

$$
A(k)=\left[\begin{array}{cc}
1 & 0.1 \\
0 & 1-0.1 \pi(k)
\end{array}\right]
$$

And $B=\left[\begin{array}{ll}0 & 0.1 o\end{array}\right]^{T}$, where $o=0.787 \mathrm{rad}^{-1} V^{-1} \mathrm{~s}^{-2}$,

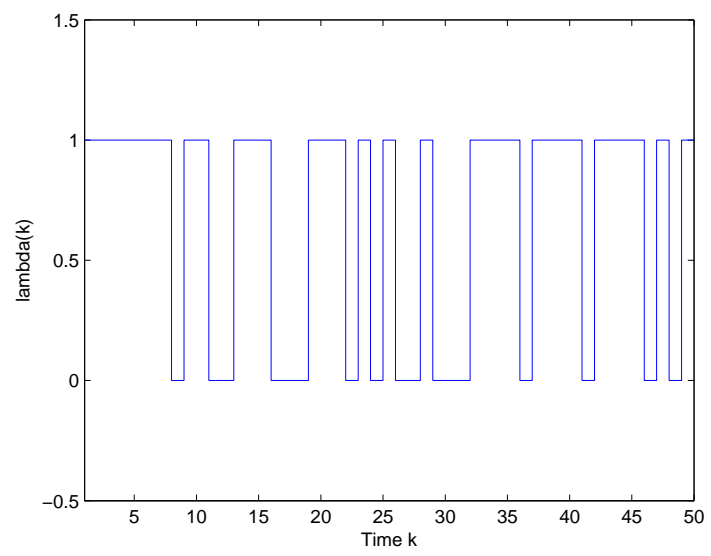

Fig. 3. data status

the parameter $\pi(k) \in[0.1,10]$ is proportional to the coefficient of viscous friction and is time varying. So $[A(k) \quad B(k)] \in \Theta=C o\left\{\left[\begin{array}{ll}A_{1} & B\end{array}\right], \quad\left[\begin{array}{ll}A_{2} & B\end{array}\right]\right\}$, and $A 1=[1,0.1 ; 0,0.99], A 2=[1,0.1 ; 0,0]$, the uncertainty set $\Theta$ is a polytope.

Here, it takes $S_{1}=I_{2}$ and $R=10^{-6}$, the forgetting factor

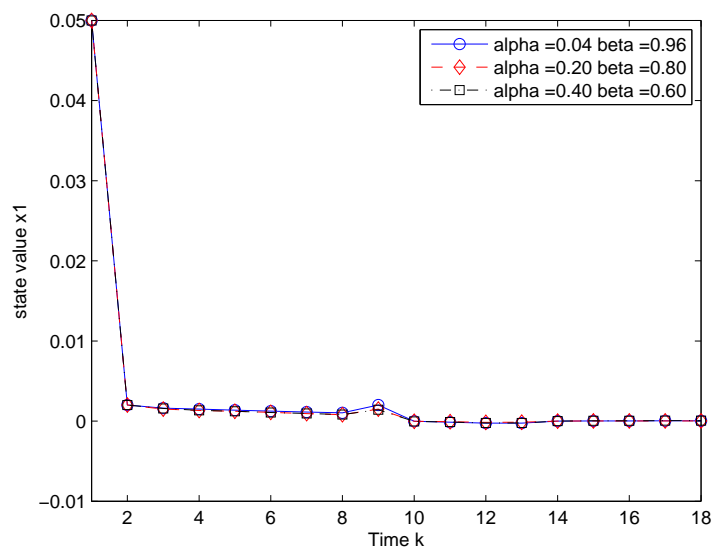

Fig. 4. system state $\mathrm{x} 1$

$\tau=0.2$ and initial value $x 0=\left[\begin{array}{ll}0.05 & 0.04\end{array}\right]^{T}, V_{\max }=2$. Then, consider the upper bound of data loss, it has $h_{\max }=3$, showing as fig 3

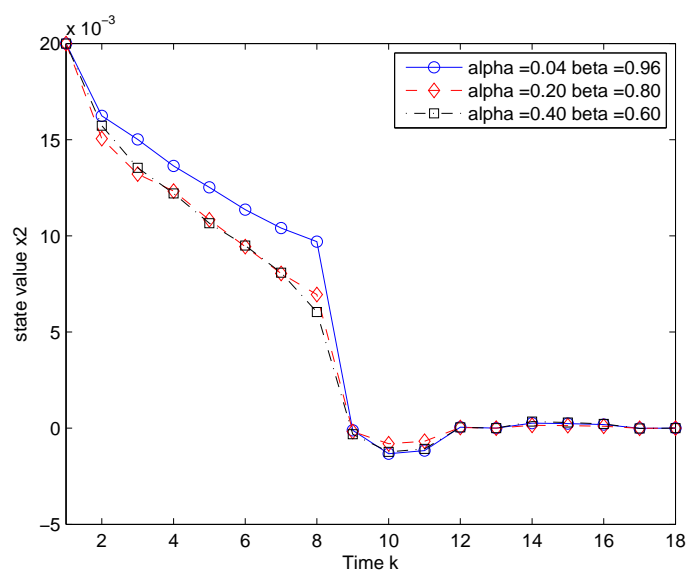

Fig. 5. system state $\mathrm{x} 2$

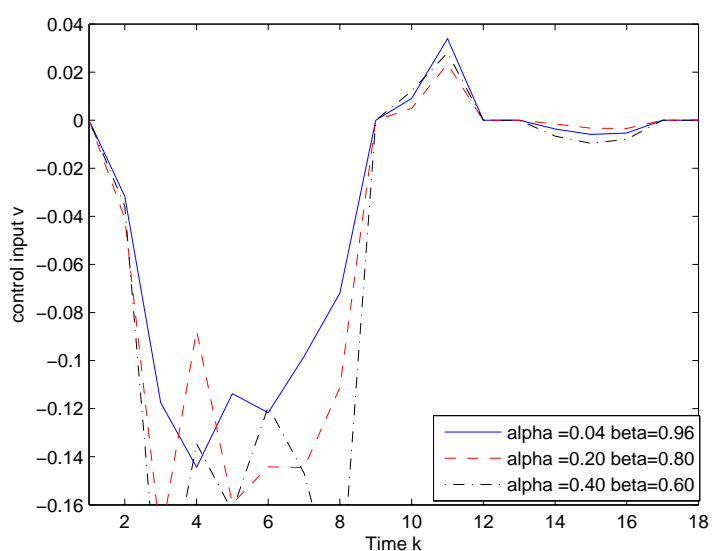

Fig. 6. control inputs $v$

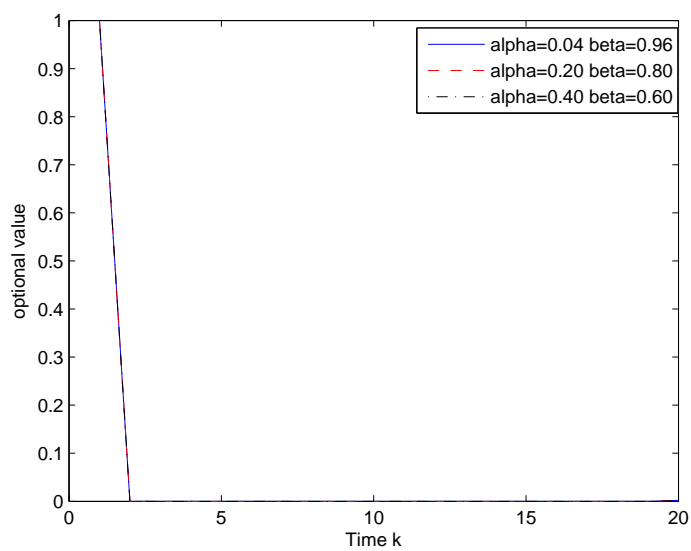

Fig. 7. option value

First, for stochastic network links, it gets the following results by proposed design consider the different missing rate $\left\{\begin{array}{ll}\alpha & \beta\end{array}\right\}$. 
Specifically, it takes $\alpha$ is $0.06,0.20,0.40$ and corresponding $\beta$ is $0.94,0.08,0.60$. Fig 4 and fig 5 are the trajectories of system

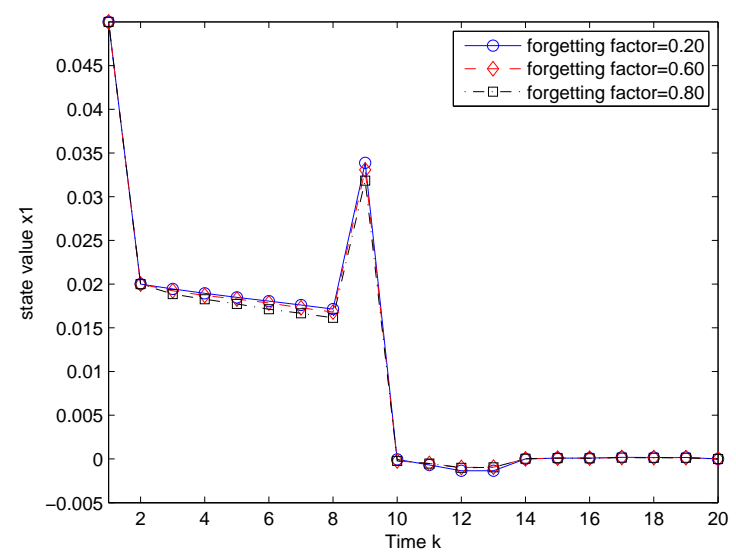

Fig. 8. system state $\mathrm{x} 1$

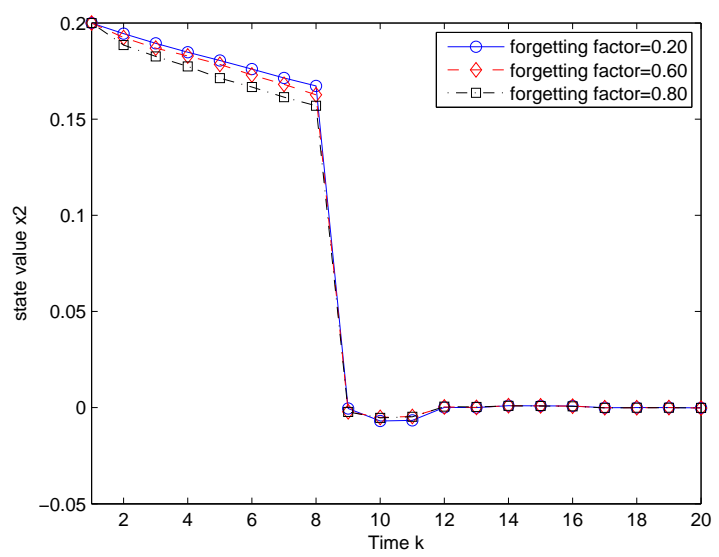

Fig. 9. system state $\mathrm{x} 2$

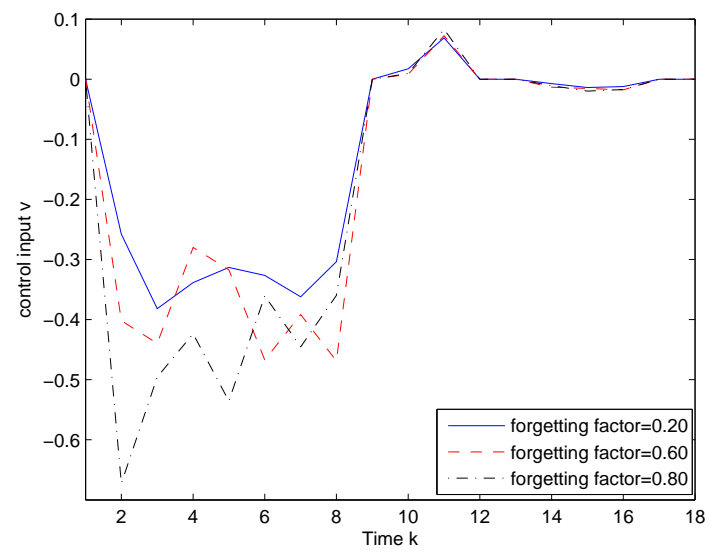

Fig. 10. control inputs $\mathrm{v}$ state $x 1, x 2$, respectively. Fig 6 is the system inputs and fig 7 is the option value $\gamma$. It is easily to see that the system state trajectories is closed to equilibrium points. So the proposed design is practical to the stochastic NCSs.

Another, the missing rate is fixed for a period of time from large scale statistics. To improve the controller performance, different forgetting factors will be selected. Here, missing rate $\alpha=0.40, \beta=0.60$. For forgetting factor $\tau$, it gives different value $0.2,0.6$ and 0.8 , therefore, the following simulation results can be obtained. Fig 8 and fig 9 represent the trajectories changes of system state $x 1, x 2$, respectively. Fig 10 is the control inputs. Thus, the system is stable.

Therefore, the following conclusions are obtained: the control law computed by this paper is effectiveness for networked control system with missing data,where missing rate is varying,furthermore, a smaller forgetting factor will improve the performance of controller,that is, the Speed of convergence of system is better.

\section{CONCLUSION}

For the stochastic-networked control system, this paper has presented a robust MPC algorithm for uncertainty models with guaranteed stochastically stable under the bounded data loss. The advantage of this algorithm is providing a state feedback law considering the four aspects as polytoic uncertainty models, stochastic invariant sets, recursive feasibility and stochastic stability. The effectiveness are shown by simulations. Furthermore, the research on robust model predictive control have remained a popular issue for networked control system closed-loop system.

\section{REFERENCES}

[1] Jianjun Bai, Hongye Su, Jinfeng Gao, and Tao Sun. Modeling and stabilization of a wireless network control system with packet loss and time delay. Journal of the Franklin Institute, 349:2420-2430, 2012.

[2] Peng $\mathrm{C}$ and Han Q. On designing a novel self-triggered sampling scheme for networked control systems with data losses and communication delays. Industrial Electronics, IEEE Transactions on, page 1, 2015.

[3] G Como and F Fagnani. Stochastic sampled-data control for synchronization of complex dynamical networks with control packet loss and additive time-varying delays. Robustness of Large-Scale Stochastic Matrices to Localized Perturbations,Network Science and Engineering, 2:53 - 64, 2015.

[4] Baocang Ding and Xubin Ping. Output feedback predictive control with one free control move for nonlinear systems represented by a takagiugeno model-fuzzy systems. IEEE Transactions on, pages 1063-6706, 2014.

[5] M Evans, M A.Cannon, and B Kouvaritakis. Robust mpc tower damping for variable speed wind turbines.control systems technology. IEEE Transactions on, pages 290 - 296, 2014.

[6] Cuzzola F.A, Geromel J.C, and Morari M. An improved approach for constrained robust model predictive control. Automatica, pages 1183-1189, 2002.

[7] J Fleming. Robust tube mpc for linear systems with multiplicative uncertainty kouvaritakis. IEEE Transactions on, pages $1087-1092,2015$. 
[8] Franze G and Tedesco F. Networked control systems: a polynomial receding horizon approach. IEEE Transactions on Control of Network Systems, 1(4):318-327, 2014.

[9] Franze G, F Tedesco, and Famularo D. Model predictive control for constrained networked systems subject to data losses. Automatica, pages 272-278, 2015.

[10] Pin G and Parisini T. Networked predictive control of uncertain constrained nonlinear systems: recursive feasibility and input-to-state stability analysis. IEEE Transactions on Automatic Control, pages 72-87, 2011.

[11] Nguyen Tuan Hung, N.Bm, Ibrahim R, and Irfan M. Design of multi model predictive control for nonlinear process plant. Intelligent and Advanced Systems (ICIAS), 2014 5th International Conference on, pages 1-6, 2014.

[12] M Killian, B Mayer, A Schirrer, and M Kozek. Cooperative fuzzy model predictive control.fuzzy systems. IEEE Transactions on, 66:1, 2014.

[13] Jiwei Li, Dewei Li, and Yugeng Xi. On design of mpc with soft constraint based on multistep control set for stochastic multiplicative system. Control Conference (CCC), pages 4102-4125, 2012.

[14] D Muoz de la Pea and P.D Christofides. Lyapunov-based model predictive control of nonlinear systems subject to data losses. IEEE Transactions on Automatic Control, 53:20762089, 2008.

[15] Kothare M.V, Balakrishnan V, and Morari M. Robust constrained model predictive control using linear matrix inequalities. Automatic, pages 1361-1379, 1996.

[16] R. Rakkiyappana. Stochastic sampled-data control for synchronization of complex dynamical networks with control packet loss and additive time-varying delays. Control Applications, 2005. CCA 2005. Proceedings of 2005 IEEE Conference on, pages $260-265,2014$.

[17] R. Rakkiyappana. Stochastic sampled-data control for synchronization of complex dynamical networks with control packet loss and additive time-varying delays. Neural Networks, 66:455-470, 2015.

[18] P.K Roy, G.K Mann, and B.C Hawlader. Fuzzy rule-adaptive model predictive control for a multivariable heating system. Neural Networks, 66:455-470, 2015.

[19] Jeong S.C and Park P. Constrained mpc algorithm for uncertain time-varying systems with state-delay. IEEE Trans. Autom. Control, pages 257-263, 2005.

[20] Nurul Adilla Mohd Subha and Guo-Ping Liu. External consensus in multi-agent systems with large consecutive data loss under unreliable networks. Neural Networks, 10(9):IET Control Theory and Applications, 2016.

[21] Haiyi Sun, QingLing Zhang, and Ning Li. Robust passive control for stochastic networked control systems. Control and Decision Conference, 2009. CCDC' '09. Chinese, pages 482 487, 2009.

[22] A Teel, A.R. Recurrence principle for stochastic difference inclusions. Automatic Control, IEEE Transactions on, 60:420 $-435,2014$.

[23] Santos T.L.M and Gonzalez A.H. Stable mpc with reduced representation for linear systems with multiple input delays. American Control Conference (ACC), pages 238-243, 2015.

[24] A.L Warren, Marlin, and Thomas E. Improved output constraint-handling for mpc with disturbance uncertainty.
American Control Conference. Proceedings of the 2003, pages $4573-4578,2003$.

[25] Hao $\mathrm{Xu}$ and S Jagannathan. Finite horizon stochastic optimal control of uncertain linear networked control system. Adaptive Dynamic Programming And Reinforcement Learning (ADPRL), 2013 IEEE Symposium on, pages 16-19, 2013.

[26] Hao Xu and S Jagannathan. Stochastic optimal controller design for uncertain nonlinear networked control system via neuro dynamic programming. Neural Networks and Learning Systems, IEEE Transactions on, 24:471 - 484, 2013.

[27] Langwen Zhang, Jingcheng Wang, Yang Ge, and Bohui Wang. Robust distributed model predictive control for uncertain networked control systems. Control Theory Applications, pages 1843-1851, 2014

[28] Lixian Zhang, Huijun Gao, and O Kaynak. Network-induced constraints in networked control systemsła survey. Industrial Informatics, IEEE Transactions on, pages 403-416, 2012.

[29] Y. Zhao, H. Gao, and T. Chen. Fuzzy constrained predictive control of non-linear systems with packet dropouts. IET Control Theory Appl, pages 1665-1677, 2010.

[30] Yuanyuan Zou, James Lam, Yugang Niu, and Dewei Li. Constrained predictive control synthesis for quantized systems with markovian data loss. Automatica, 0:217-225, 2015. 